\title{
Complete valence-band structure of Ge determined by photoemission
}

\author{
X. H. Chen, W. Ranke, and E. Schröder-Bergen \\ Fritz-Haber-Institut der Max-Planck-Gesellschaft, Faradayweg 4-6, D-1000 Berlin 33, West Germany
}

(Received 1 June 1990)

\begin{abstract}
The valence-band structure along the high-symmetry directions $\Gamma L, \Gamma X$, and $\Gamma K X$ was studied with synchrotron radiation for a wide photon energy range (10-106 eV). A cylindrically shaped Ge crystal with [110] axis was used, which allowed us to record the spectra in normal emission for different orientations. The data were analyzed by applying the model of direct transition into freeelectron final-state bands. Due to the wide photon energy range, a complete band scheme along the three directions including critical points was obtained. The data are compared with theoretical results and other measurements. The bands along the $\Gamma K X$ direction are reported for the first time to our knowledge. Applying the wide photon energy range, the inner potential $V_{0}=-8.8 \mathrm{eV}$ was determined self-consistently using the method suggested by Middelmann et al. [Phys. Rev. B 34, 957 (1986)].
\end{abstract}

\section{INTRODUCTION}

The bulk valence-band structure of germanium has been studied both experimentally and theoretically. ${ }^{1-7}$ Three angular-resolved photoemission studies on $\mathrm{Ge}(001)(2 \times 1)$ have been carried out to map the band dispersions along the $\Gamma X$ direction. ${ }^{1-3}$ Despite differences in the identifications of surface states, the studies show good general agreement for the bulk bands with the theoretical calculation of Chelikowsky and Cohen. ${ }^{4}$ The band dispersions along the direction $\Gamma L$ have also been studied on (111) samples by two groups. ${ }^{5,6}$ Either calculated final state bands or a free-electron-like final-state band have been used to interpret the results on $\mathrm{Ge}(111)(2 \times 1)$ and $\mathrm{Ge}(111) c(2 \times 8)$, respectively. For the photon energy range between about 50 and $70 \mathrm{eV}$, Wachs et al. ${ }^{5}$ explain their data by assuming transitions to primary-cone free-electron bands. In the photon energy range below $30 \mathrm{eV}$, the situation seems different, and $\mathrm{Ni}$ cholls et al. ${ }^{6}$ find no indication for such transitions. No measurement of the bulk valence band along the direction $\Gamma K X$ has been performed so far.

In this paper, we present band-mapping measurements by angle-resolved ultraviolet photoemission spectroscopy of the bulk valence bands along the symmetry directions $\Gamma K X, \Gamma L$, and $\Gamma X$ on a cylindrical Ge sample with [1 10$]$ as cylinder axis. The cylinder surface displays (110), (111), and (001) orientations. Simple rotation of the sample allows us thus to measure in situ the normal emission from these high-symmetry orientations. This makes data analysis easy because the direction of the momentum vector $\mathbf{k}$ is conserved in the emission process.

We apply a free-electron approximation for the finalstate dispersion. The wide photon energy range used allows us to determine not only the bulk dispersions in a wide range in $\mathbf{k}$ space but also the inner potential $V_{0}$ by applying the method suggested by Middelmann et al. ${ }^{8}$

Our experimental dispersion along $\Gamma L$ and $\Gamma X$ is in good agreement with existing calculations and the experiments published so far. ${ }^{1-7}$ However, due to our wide photon energy range, we present a complete determination in both directions. The experimental dispersions along $\Gamma K X$ are reported for the first time, to our knowledge. The paper is organized as follows. The apparatus and sample preparation are briefly described in Sec. II. In Sec. III we present results and discussion, first for $\mathrm{Ge}(110)$, then for (001) and (111). The conclusion is given in Sec. IV.

\section{EXPERIMENT}

The angle-resolved photoemission experiment was performed in an ADES 400 instrument from Vacuum Generators with angle resolution $\pm 1.5^{\circ}$. Using the toroidal grating monochromator TGM3 at the Berlin Storage Ring (BESSY), a photon energy range from 10 to $106 \mathrm{eV}$ was covered. The overall energy resolution was between $0.2 \mathrm{eV}$ for the lower photon energies and $0.5 \mathrm{eV}$ at the highest photon energies. The binding energies refer to the valence-band maximum (VBM). This level was determined by assuming a value of $29.3 \mathrm{eV}$ for the $\mathrm{Ge} 3 d_{5 / 2}$ binding energy. ${ }^{9}$

A cylindrically shaped Ge sample, as described in detail before, ${ }^{10}$ was used to study the bulk valence bands along the $\Gamma L, \Gamma X$, and $\Gamma K X$ directions. The cylinder axis was [1이, so that the photoemission of the lowindex orientations (110), (111), and (001) as well as all intermediate orientations of the $[1 \overline{1} 0]$ zone could be investigated in normal emission. The incidence angle of light was always $45^{\circ}$ with respect to the surface normal. The cylinder had a diameter of $24 \mathrm{~mm}$ and was ring shaped with the sample heater in its center. Due to the width of the light beam, the orientation resolution was about $3^{\circ}$. The surface was cleaned by cyclic sputtering $(U=1 \mathrm{kV})$ and annealing $(T=900 \mathrm{~K})$. The cleanliness and order on the surface was checked by ultraviolated photoemission spectroscopy and low-energy electron diffraction, respectively. 


\section{RESULTS AND DISCUSSION}

Figure 1 shows selected normal emission spectra of $\mathrm{Ge}(110) c(8 \times 10)$ for a photon energy range $h v=12-100$ $\mathrm{eV}$. The peak labeled $A$ for $h v=35.9 \mathrm{eV}$ is the Ge $M V V$ Auger transition. Peak $B$ for $h v=18-24 \mathrm{eV}$ is the Ge $3 d$ peak generated by the second-order light of the monochromator spectrum. Both peaks mask the valence-band emission in the (not shown) photon energy range from 24 to $35 \mathrm{eV}$. The features $C-E$ in the spectra vary as expected for dispersing bands. Features $F-I$ have a constant binding energy. In order to assign all features, we assume direct transition into free-electron final bands with a dispersion $E_{f}=E\left(\mathbf{k}_{f}\right)$ given by

$$
E_{f}\left(\mathbf{k}_{f}\right)=\left[\hbar^{2}\left(\mathbf{k}_{f}+\mathbf{G}\right)^{2} / 2 m\right]+V_{0},
$$

where $\mathbf{k}_{f}$ is the electron momentum perpendicular to the surface, $V_{0}$ is the inner potential referring to the VBM and $\mathbf{G}$ is a bulk reciprocal-lattice vector which is involved in the photoemission process (bulk umklapp). Transitions with $\mathbf{G}$ in the same direction as $\mathbf{k}_{l}$ and thus also $\mathbf{k}_{f}$ and for normal emission (primary-cone emission) are easy to analyze and represent the main structures in the spectra, as will be shown. Emission with $\mathbf{G}$ not per-

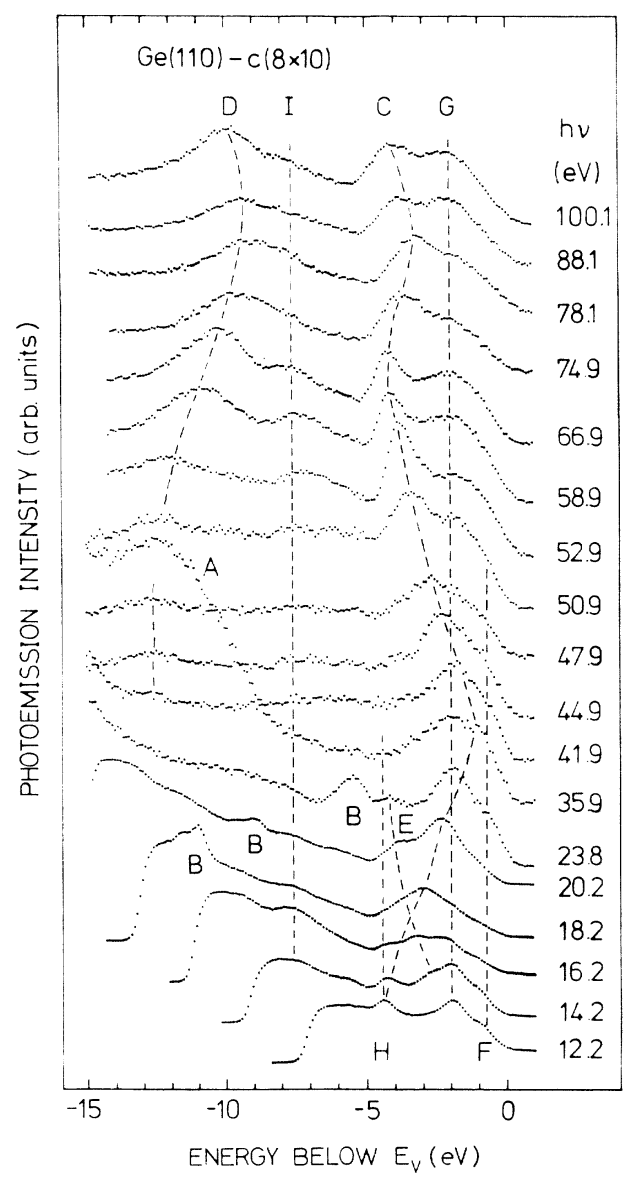

FIG. 1. Selected normal-emission spectra for Ge(110) recorded with the indicated photon energies. $A$, Auger $M V V$ peak; $B$, Ge $3 d$ emission by second-order light of the grating; $C-I$, features from bulk and surface-state emission (see text). pendicular to the surface (called secondary-cone emission) leads to less intense structures in the spectra.

Assuming primary-cone emission for all features in Fig. 1, the corresponding initial states were calculated and are presented in Fig. 2. For some points, error bars are given, representing the uncertainty of $\pm 0.15 \mathrm{eV}$ for sharp structures and $\pm 0.5 \mathrm{eV}$ for broad and weak structures. The solid lines are theoretical valence bands, as calculated by Chelikowsky and Cohen using a nonlocal pseudopotential method. ${ }^{4}$ There are altogether four valence bands, labeled $1-4$ in Fig. 2. The dispersive peaks $C$ and $D$ in Fig. 1 are assigned to direct transitions from bands 1 and 3, respectively. These two experimental bands show a good general agreement with the corresponding theoretical bands. Bands 2 and 4 were hardly observable. The related spectra for photon energies from 12 to $23 \mathrm{eV}$ in Fig. 1 had three nondispersive peaks $G, H$, and $I$, centered around $-2.0,-4.3$, and $-7.6 \mathrm{eV}$, respectively, which will be discussed below. The expected dispersive peaks corresponding to bands 2 and 4 run rapidly through these three peaks and are obviously weak, so that it was difficult to distinguish them.

Peak $E$ in Fig. 1 seems also dispersive. However, the corresponding points labeled $E$ in Fig. 2 do not fit with an expected band. We believe that these transitions are of the secondary-cone type. Both transitions from band 2

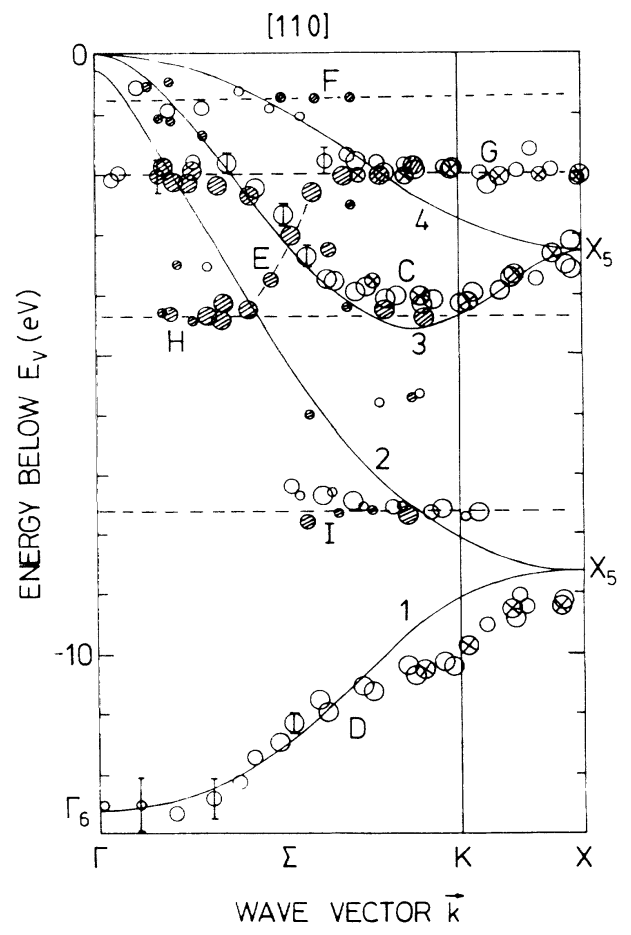

FIG. 2. Experimentally determined points and theoretical bands (solid lines, from Ref. 4) of Ge along the $\Gamma K X$ direction. Large circles denote strong peaks. The differently marked circles are for different photon energy ranges, and different reciprocal-lattice vectors are involved in the transition to the free-electron final band: shaded circles for low $h v$ and $G_{\overline{22} 0}$, open circles for medium $h v$ and $G_{220}$, and circles with crosses for large $h v$ and $G_{\overline{4} \overline{4} 0}$. Some critical points are also labeled. Features labeled $C-I$ correspond to the peaks in Fig. 1 . 
involving the reciprocal-lattice vector $G_{020}$ from band 3 involving $G_{111}$ could be responsible.

In the present analysis an inner potential of $V_{0}=-8.8$ $\mathrm{eV}$ with respect to the VBM was assumed. The general shape of the bands and their absolute position are not very sensitive to the choice of $V_{0}$ and different values between $-7.7,{ }^{2,5}-8.0,{ }^{3}-8.8,{ }^{11}$ and $-9.5 \mathrm{eV}^{6}$ were used before. Middelmann et al. ${ }^{8}$ have described a method to determine $V_{0}$ self-consistently, if a sufficiently large photon energy range is available. In this case, the same initial state can sometimes be excited into successive freeelectron final bands involving different reciprocal-lattice vectors. For example, the peak labeled $C$ in Fig. 1 runs from near $\Sigma_{\min }$ for $h v=12 \mathrm{eV}$ (minimum of band 3) towards $\Gamma(h v=36 \mathrm{eV})$, back to $\Sigma_{\min }(h v=60 \mathrm{eV})$, up towards $X_{5}(h v=78 \mathrm{eV})$, and back towards $\Sigma_{\text {nin }}(h v \geq 100$ $\mathrm{eV}$ ). Peak $D$ (band 1) has reached $X_{5}$ for $h v=85 \mathrm{eV}$ and turns back with increasing $h v$. To illustrate this better, the points in Fig. 2 originating from different ranges of $h v$ and involving different primary-cone reciprocal-lattice vectors are distinguished by different symbols. The inner potential has to be chosen so that all band branches for different ranges of $h v$ yield the same initial band. The best fit was obtained for $V_{0}=-8.8 \pm 0.5 \mathrm{eV}$.

Besides the dispersive peaks, there exist also the peaks $F, G$, and $H$, and $I$ in Fig. 1, which are sometimes weak but if visible appear always at the same position. They represent either surface states or emission from regions with a high density of states implying secondary-cone transitions, scattering by defects, or other kinds of indirect transitions. We assign the features around -7.6 and $-4.3 \mathrm{eV}$ to critical points at $L_{6}$ (see below and Fig. 4) and $\Sigma_{\min }$, respectively. Since there exist no corresponding critical points around -2.0 and $-0.8 \mathrm{eV}$, we assume that these peaks represent surface states. This assignment could be examined by gas adsorption.

The normal emission spectra of $\mathrm{Ge}(001)(2 \times 1)$ and $\mathrm{Ge}(111) c(2 \times 8)$ were also recorded to determine the bulk valence bands along the directions $\Gamma X$ and $\Gamma L$. Figure 3 shows selected spectra from $\operatorname{Ge}(001)(2 \times 1)$ for $h v$ $=12-88 \mathrm{eV}$. The spectra are in good agreement with those published before. $^{1-3}$ Also spectra of $\mathrm{Ge}(111) c(2 \times 8)$, which are not shown here, agree well with those reported by Wachs et al. ${ }^{5}$ and Nicholls et al. ${ }^{6}$ The same analysis as for $\mathrm{Ge}(110)$ yields the points displayed in Fig. 4. The solid lines labeled 1, 2, 3, and 4 indicate the calculated valence-band dispersions of $\mathrm{Ge}$ from Alekseev et al. using the linearized augmented plane-wave method with virtual spheres. ${ }^{7}$ The energy bands are somewhat wider and show a better agreement with the results of spectroscopic experiments than those calculated by Chelikowsky and Cohen. ${ }^{4}$ Since the spinorbit interaction was not considered in the calculation of Alekseev et al., bands- 3 and 4 are not split along the $\Gamma L$ direction.

Peak $C$ in Fig. 3 is assigned to transitions from bands 3 and 4 , and peak $D$ to bands 1 and 2 in the $\Gamma X$ direction. Band 1 along the $\Gamma X$ line, corresponding to the spectra for $h v=44.9-88.1 \mathrm{eV}$ in Fig. 3, is fully determined due to our wide photon energy range. The agreement with the theoretical results of Alekseev et al. ${ }^{7}$ is good. Only the assignment of the peaks with low binding energy around -0.5 and $-1.3 \mathrm{eV}$ corresponding to peaks labeled $F$ and $G$ for photon energy below $18.2 \mathrm{eV}$ in Fig. 3 is ambiguous. Hsieh et al. ${ }^{2}$ considered these two states as bulk states, whereas they were ascribed to surface states by Nelson et al. ${ }^{1,12}$ The controversy seems to be resolved by Krüger et al. ${ }^{13}$ Using self-consistent scattering calculations, they found that two surface states, corresponding to a dangling bond state on the raised dimer atom and a back-bond state, have the measured energy positions. The dangling-bond state becomes a very broad and weak resonance at the $\Gamma$ point. Following this explanation, we believe that the bulk contribution dominates in the sharp features below $h v=16.2 \mathrm{eV}$. However, admixture of surface-state emission may influence the peak positions.

The position of peak $E$ varies with photon energy. The evaluated sequence, shown labeled $E$ along $\Gamma X$ in Fig. 4, does not agree with an expected band. Like peak $E$ in Fig. 1, it originates probably from secondary-cone processes.

Our experimental results on $\mathrm{Ge}(111) c(2 \times 8)$ along $\Gamma X$ are also included in Fig. 4. The experimental bands are

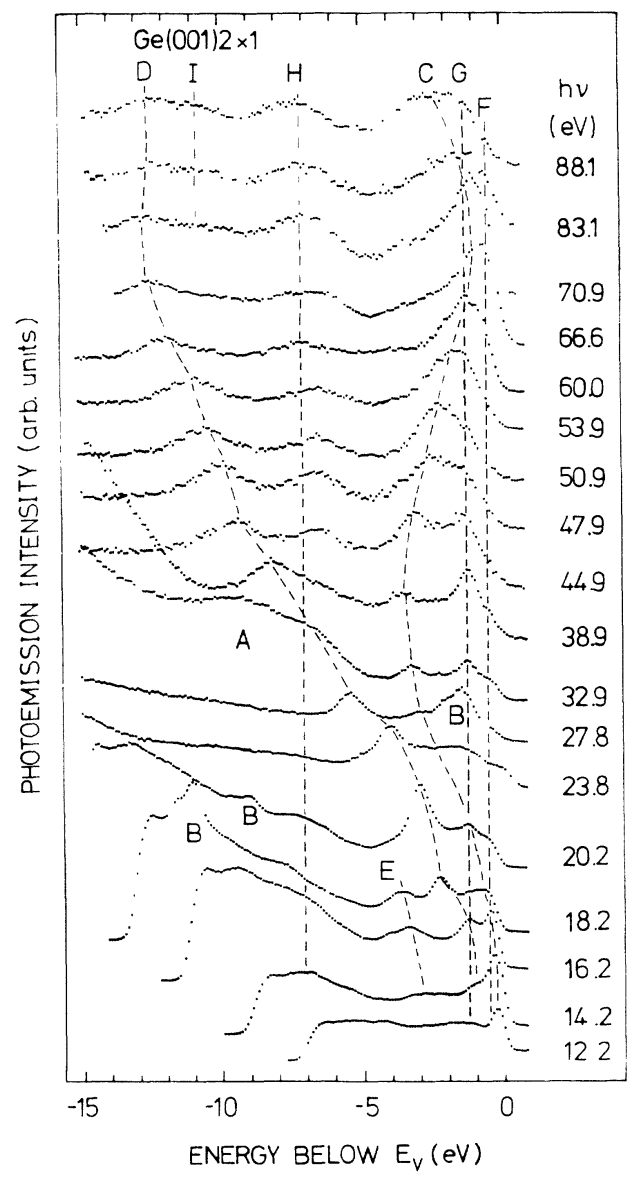

FIG. 3. Selected normal-emission spectra for Ge(001) $2 \times 1$ recorded with the indicated photon energies. Peak labeling as in Fig. 1. 


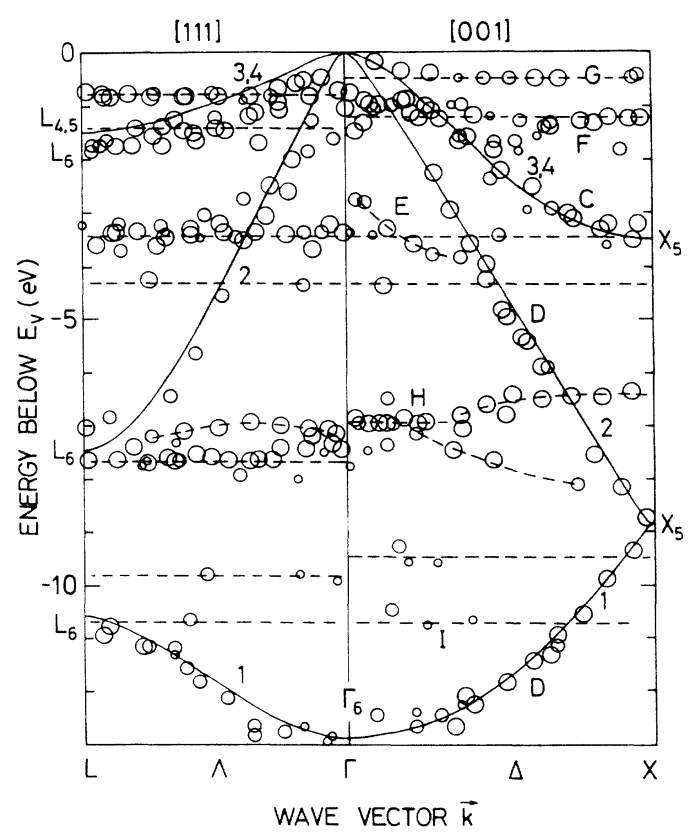

FIG. 4. Experimentally determined points and theoretical bands (solid lines, from Ref. 7) of $\mathrm{Ge}$ along the $\Gamma X$ and $\Gamma L$ directions. Large circles denote strong peaks. Features labeled $C-I$ in the $\Gamma X$ direction correspond to the peaks in Fig. 3.

also in general agreement with the theory ${ }^{4,7}$ and with the experimental results of Wachs et al. ${ }^{5}$ Only band 3 shows a systematic deviation from the calculated band. As in the results of Wachs et al. ${ }^{5}$ only the spectra for a photon energy above about $50 \mathrm{eV}$ contribute to dispersing bands. The spectra for smaller photon energies are dominated by nondispersive features which cannot be used for band mapping in a simple way. A detailed analysis of the spectra between 10 and $30 \mathrm{eV}$, recorded with the polarized light, was made by Nicholls et al. ${ }^{6}$ They also do not observe transitions to the primary (111) free-electron band in normal-emission spectra.

As for the spectra of $\mathrm{Ge}(110)$, there exist also nondispersive peaks along the directions $\Gamma L$ and $\Gamma X$, associated with surface states or critical points in the valenceband structures with a high density of states, as discussed

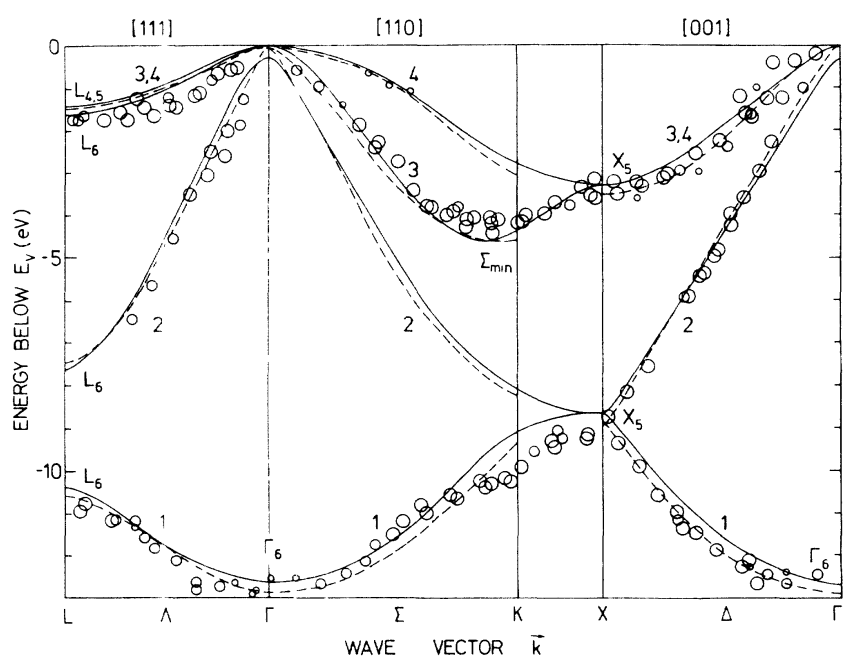

FIG. 5. Experimental (circles) and theoretical (solid and dashed lines) band structures of $\mathrm{Ge}$ along the $\Gamma L, \Gamma X$, and $\Gamma K X$ directions. The solid and dashed lines indicate the calculated bands from Refs. 4 and 7, respectively.

above. Along $\Gamma L$ in Fig. 4 , two features at -0.8 and $-1.4 \mathrm{eV}$ were assigned to surface states. The latter appears clearly in the spectra for photon energies below 28 $\mathrm{eV}$ and becomes weak for higher photon energy. Since the overlap with peaks of bands 3 and 4 was encountered only for high photon energies, it was not difficult to distinguish them from each other. The third surface state, reported by Aarts et al. ${ }^{14}$ at $-0.15 \mathrm{eV}$ was not observed by us. Most of the other undispersive features correspond to critical points. For example, the feature around -3.4 along the direction $\Gamma L$ in Fig. 4 coincides with point $X_{5}$. The peaks at $-4.3 \mathrm{eV}$ in both directions could be from point $\Sigma_{\min }$ in the $\Gamma K X$ direction. Point $L_{6}$ of band 2 contributes to the features around $-7.6 \mathrm{eV}$ along the $\Gamma L$ direction, which showed some small shifts with photon energy. In contrast to results published before, ${ }^{1,2}$ the features $H$ around $-7.0 \mathrm{eV}$ along the $\Gamma X$ direction, which are slightly dispersing when approaching $X$, as well as the slightly dispersing band at $-7.0 \mathrm{eV}$ in the $\Gamma L$ direction, do not seem to relate to point $L_{6}$ because of the

TABLE I. Experimental and theoretical energy values of different bands at critical and other special points along the $\Gamma L, \Gamma X$, and $\Gamma K X$ directions, referring to the VBM in $\mathrm{eV}$.

\begin{tabular}{lccccc}
\hline \hline & Point & $\begin{array}{c}\text { This work } \\
\text { (experiment) }\end{array}$ & $\begin{array}{c}\text { Ref. 7 } \\
\text { (theory) }\end{array}$ & $\begin{array}{c}\text { Ref. 4 } \\
\text { (theory) }\end{array}$ & $\begin{array}{c}\text { Ref. 15 } \\
\text { (experiment) }\end{array}$ \\
\hline Band 1 & $\Gamma_{6}$ & $-12.9 \pm 0.5$ & -12.9 & -12.66 & $-12.6 \pm 0.3$ \\
& $L_{6}$ & $-10.7 \pm 0.4$ & -10.6 & -10.39 & $-10.6 \pm 0.5$ \\
Band 2 & $K$ & $-10.1 \pm 0.2$ & -9.25 & -9.1 & \\
Bands 1,2 & $L_{6}$ & $-7.6 \pm 0.2$ & -7.4 & -7.61 & $-7.7 \pm 0.2$ \\
Band 3 & $X_{5}$ & $-8.8 \pm 0.2$ & -8.9 & -8.65 & \\
& $L_{6}$ & $-1.8 \pm 0.4$ & -1.5 & -1.63 & $-1.4 \pm 0.3$ \\
Band 4 & $\Sigma_{\min }$ & $-4.3 \pm 0.2$ & & -4.6 & $-4.5 \pm 0.2$ \\
Bands 3,4 & $K$ & $-4.2 \pm 0.2$ & -4.6 & -4.35 & $-1.4 \pm 0.3$ \\
\hline \hline
\end{tabular}


large energy value difference. We could not assign these features till now. The shifts with photon energy could be a hint for secondary-cone processes. The weak peaks around $-10.7 \mathrm{eV}$ in the $\Gamma L$ and $\Gamma X$ directions can arise from point $L_{6}$ of band 1 . For the other weak features between -9 and $-10 \mathrm{eV}$ we have no reasonable explanation.

\section{CONCLUSION}

As a summary, we present the complete valence-band structure for Ge in Fig. 5. All peaks which do not correspond to primary-cone bulk band emission are omitted. The band dispersions from calculations of Chelikowsky and Cohen $^{4}$ and Alekseev et al. ${ }^{7}$ are indicated by solid and dashed lines, respectively. Our results show a better agreement with the calculation of Alekseev et al. Due to our wide photon energy range, the critical points could be determined relatively accurately. In order to compare with the theoretical results ${ }^{4,7}$ and other measurements, ${ }^{15}$ these critical points are summarized in Table I. We cannot resolve the separation between points $L_{6}$ and $L_{4,5}$ of bands 3 and 4 that are induced by the spin-orbit interaction. Apart from a distinct deviation at these two points, our results show a generally good agreement.

\section{ACKNOWLEDGMENTS}

The cooperation of and stimulating discussion with $\mathrm{U}$. Myler, K. Jacobi, and P. Geng are gratefully acknowledged. One of us (X.H.C.) was supported by the Technische Universität Berlin and one of us (E.S.) by the Sonderforschungsbereich 6 der Deutschen Forschungsgemeinschaft.
1J. G. Nelson, W. J. Gignac, R. S. Williams, S. W. Robey, J. G. Tobin, and D. A. Shirley, Surf. Sci. 131, 290 (1983).

${ }^{2}$ T. C. Hsieh, T. Miller, and T.-C. Chiang, Phys. Rev. B 30, 7005 (1984).

${ }^{3}$ J. H. Neave, P. K. Larsen, B. A. Joyce, J. P. Gowers, and J. F. Van der Veen, J. Vac. Sci. Technol. B 1, 668 (1983).

${ }^{4}$ J. R. Chelikowsky and M. L. Cohen, Phys. Rev. B 14, 556 (1976).

${ }^{5}$ A. L. Wachs, T. Miller, T. C. Hsieh, A. P. Shapiro, and T.-C. Chiang, Phys. Rev. B 32, 2326 (1985).

${ }^{6}$ J. M. Nicholls, G. V. Hansson, U. O. Karlsson, P. E. S. Persson, R. I. G. Uhrberg, R. Engelhardt, S. A. Flodström, and E.-E. Koch, Phys. Rev. B 32, 6663 (1985).

${ }^{7}$ E. S. Alekseev, L. B. Litinskii, and A. I. Likhter, Fiz. Tech. Poluprovodn. 22, 2059 (1988) [Sov. Phys. Semicond. 22, 1303 (1988)].
${ }^{8}$ H. U. Middelmann, L. Sorba, V. Hinkel, and K. Horn, Phys. Rev. B 34, 957 (1986).

${ }^{9}$ H. J. Kuhr and W. Ranke, Solid State Commun. 61, 285 (1987).

${ }^{10}$ H. J. Kuhr and W. Ranke, Surf. Sci. 187, 98 (1987).

${ }^{11}$ D. Straub, L. Ley, and F. J. Himpsel, Phys. Rev. B 33, 2607 (1986).

12J. G. Nelson, W. J. Gignac, R. S. Williams, S. W. Robey, J. G. Tobin, and D. A. Shirley, Phys. Rev. B 27, 3924 (1983).

${ }^{13}$ P. Krüger, A. Mazur, J. Pollmann, and G. Wolfgarten, Phys. Rev. Lett. 57, 1468 (1986).

${ }^{14}$ J. Aarts, A. J. Hoeven, and P. K. Larsen, Phys. Rev. B 37, 8190 (1988).

${ }^{15}$ D. E. Eastman, W. D. Grobman, J. L. Freeouf, and M. Erbudak, Phys. Rev. B 9, 3473 (1974); W. D. Grobman, D. E. Eastman, and J. L. Freeouf, Phys. Rev. B 12, 4405 (1975). 